\title{
LiveSync: Deformed Viewing Spheres for Knowledge-Based Navigation
}

\author{
Peter Kohlmann, Stefan Bruckner, Armin Kanitsar and M. Eduard Gröller, Member, IEEE
}

\begin{abstract}
Although real-time interactive volume rendering is available even for very large data sets, this visualization method is used quite rarely in the clinical practice. We suspect this is because it is very complicated and time consuming to adjust the parameters to achieve meaningful results. The clinician has to take care of the appropriate viewpoint, zooming, transfer function setup, clipping planes and other parameters. Because of this, most often only 2D slices of the data set are examined. Our work introduces LiveSync, a new concept to synchronize 2D slice views and volumetric views of medical data sets. Through intuitive picking actions on the slice, the users define the anatomical structures they are interested in. The 3D volumetric view is updated automatically with the goal that the users are provided with expressive result images. To achieve this live synchronization we use a minimal set of derived information without the need for segmented data sets or data-specific pre-computations. The components we consider are the picked point, slice view zoom, patient orientation, viewpoint history, local object shape and visibility. We introduce deformed viewing spheres which encode the viewpoint quality for the components. A combination of these deformed viewing spheres is used to estimate a good viewpoint. Our system provides the physician with synchronized views which help to gain deeper insight into the medical data with minimal user interaction.
\end{abstract}

Index Terms—Navigation, interaction, linked views, medical visualization, viewpoint selection.

\section{INTRODUCTION}

Modern modalities for medical imaging (e.g., computed tomography) provide large quantities of data at an unprecedented resolution. Presenting this enormous amount of information is a challenging task for today's radiology workstations. Volumetric rendering is the current method of choice for providing a good survey of the data. Combining the information provided by two-dimensional cross-sections and threedimensional visualization can improve the diagnosis process. Linking the different representations of the data has the potential benefit to provide significant enhancements in efficiency. Usually the volumetric display acts as an overview display in this context. The cross-sectional images contain diagnostically relevant information.

Pinpointing a pathological area in the volumetric display selects the corresponding cross-sectional images to be displayed in the twodimensional display area. From a technical point of view this process is relatively easy to implement. The $3 \mathrm{D}$ position of the interesting point can be deduced from the given viewport specification (i.e., transfer function and viewing direction). Very important to note is the reduced degree of freedom in highlighting the position on the corresponding cross-sectional image. The reverse operation is, however, not that straightforward. Picking a $2 \mathrm{D}$ position on a cross-sectional slice should result in an expressive unobstructed 3D view. Even though the interesting position is well defined by selecting a point in the crosssectional image, the appropriate highlighting of the area of interest in $3 \mathrm{D}$ is challenging. The general motivation for emphasizing a structure selected in 2D in its three-dimensional setting is to get the contextual information. A short example illustrates the situation: A frequently occurring request during reading cross-sectional images of computed tomography angiography is to determine to which anatomical structure a specific partially visible vessel belongs. In this case a volumetric rendering of the depicted vessel and its spatial vicinity would be desired. For optimal results the selected structure should be visible to a large

- Peter Kohlmann, Stefan Bruckner and M. Eduard Gröller are with the Institute of Computer Graphics and Algorithms, Vienna University of Technology, E-mail: \{kohlmann_bruckner_groeller\}@cg.tuwien.ac.at.

- Armin Kanitsar is with AGFA HealthCare, E-mail: armin.kanitsar@agfa.com.

Manuscript received 31 March 2007; accepted 1 August 2007; posted online 27 October 2007.

For information on obtaining reprints of this article, please send e-mail to: tvcg@computer.org. extent and must not be occluded by structures of lower importance.

In the following, a novel concept for interactive viewpoint estimation based on a limited set of input parameters, preserving the generality of the approach, is presented. The only necessary user interaction to derive all the input parameters is given by the picking of a position on a slice. A viewing sphere surrounds the center of a scanned data set and describes all possible camera positions with respect to this object. The input parameters are utilized to encode viewpoint quality in deformed viewing spheres whenever a picking action is performed. After combining the deformed spheres for the different parameters, the estimated quality for all possible viewpoints on the picked structure of interest can be determined from the resulting sphere.

This paper is structured as follows: Section 2 provides an overview on the relevant previous work. In Section 3 , the workflow and the input parameters are described. Section 4 introduces the concept of the viewing sphere. In Section 5, it is shown how the input parameters are utilized for the deformation of the viewing sphere. The weighing of the spheres for the different parameters and the combination operators are described in Section 6. Section 7 explains how the viewing setup can be arranged with the derived viewport parameters. In Section 8 , LiveSync-generated images are presented for different data sets and the results of an informal evaluation are summarized. Finally, Section 9 concludes the paper and indicates directions for future research.

\section{Related Work}

Viewpoint selection is a well investigated research area for polygonal scenes but relatively few research has been done in the scope of volumetric data. Moreover, the combination of optimal viewpoint estimation and synchronized views has received little attention within the community.

Fleishman et al. [5] presented an approach for an automatic placement of the camera for image-based models with known geometry. A quality measure is applied for the visibility and the occlusion of surfaces. Methods like canonical views are investigated by Blanz et al. [1] for aesthetic aspects of a viewpoint. In their experimental setup users assign goodness ratings to viewpoints for three-dimensional object models. Based on the feedback a set of criteria for good viewpoints is defined. To determine the viewpoint quality for virtual scenes Sbert et al. [11] applied a measure based on the Kullback-Leibler distance of the projected area of the polygons in the scene. The mesh saliency approach introduced by Lee et al. [6] measures a regional importance for meshes. Besides mesh simplification this can be employed for viewpoint selection as well. Vázquez et al. [15, 16] worked 


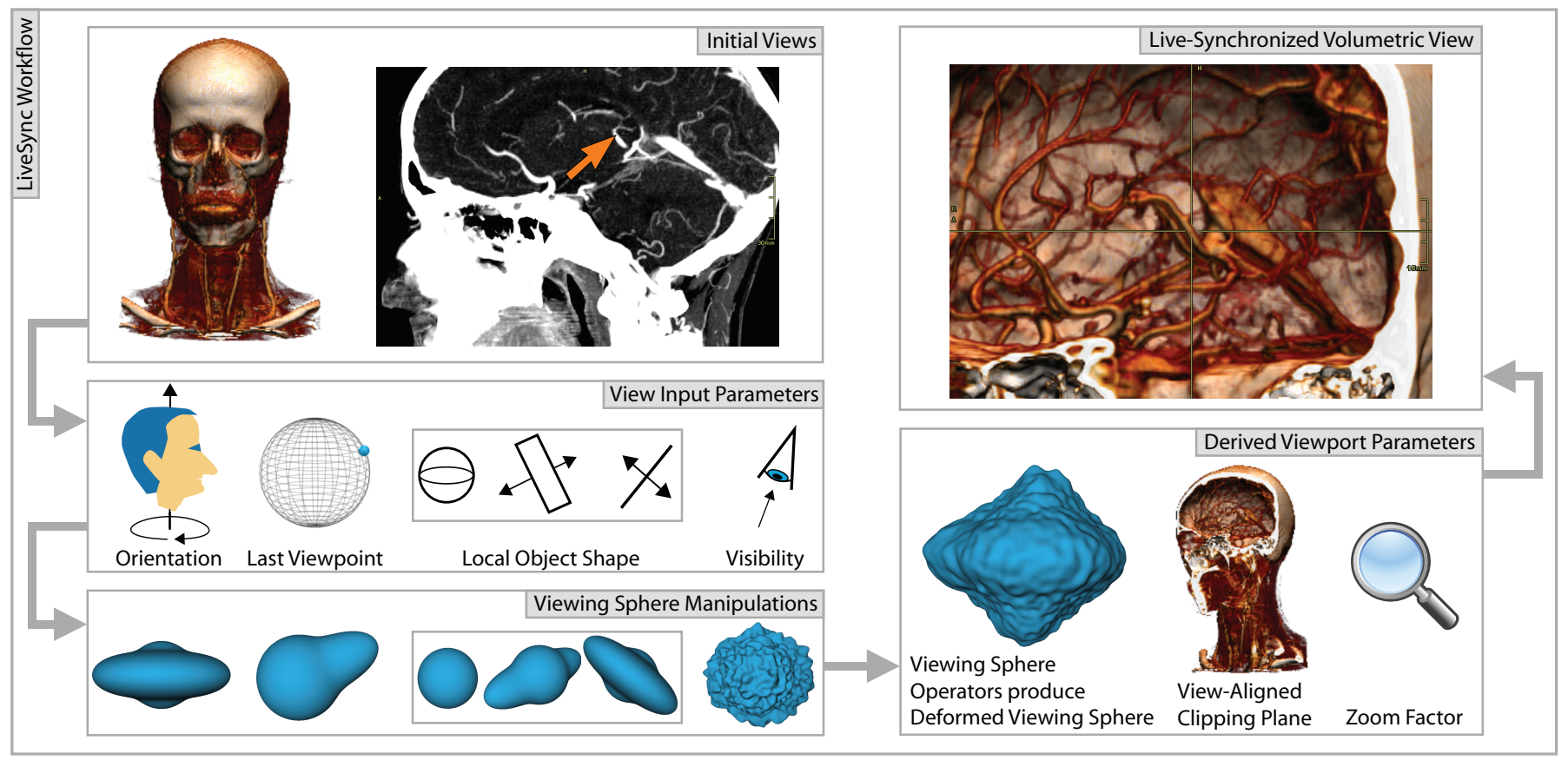

Fig. 1. LiveSync workflow: Initially there is a volumetric view and a 2D slice image. A picking action on the slice initiates the deformation of viewing spheres for the input parameters: patient orientation, viewpoint history, local shape estimation, and visibility. The combination of these parameters leads to a single deformed viewing sphere which encodes the quality of the viewpoints. In addition, a view-aligned clipping plane is positioned and the zoom is adjusted. These steps generate a live-synchronized volumetric view which provides a good view on the picked structure.

on the problem that in computer graphics there is no consensus about what defines a good view. Viewpoint entropy based on information theory is introduced to compute good viewing positions automatically. Polonsky et al. [9] aimed for the computation of the best view of an object. They define a set of view descriptors to measure the viewpoint quality. Mühler et al. [7] presented an approach for viewpoint selection in medical surface visualizations. Their work aims at the generation of animations for collaborative intervention planning and surgical education.

Inspired by the research work on polygonal data there is some recent work on viewpoint selection for volumetric data. Bordoloi and Shen [2] presented an entropy-based approach to determine a minimal set of representative views for a given scene. The data distribution, the transfer function and the visibility of voxels are taken into account for their viewpoint selection process. A feature-driven approach to select a good viewpoint is proposed by Takahashi et al. [12]. They identified feature components in the volume for the detection of locally optimal viewpoints. These viewpoints are utilized to extract an optimal global viewpoint. Viola et al. [17] introduced an importance-driven approach to focus on structures within volumetric data. The focus object is defined by the user and their system automatically selects a characteristic viewpoint which provides an expressive view on the object of interest. A framework which facilitates viewpoint selection for angiographic volumes is presented by Chan et al. [4]. View descriptors for visibility, coverage and self-occlusion of the vessels are considered to determine a globally optimal view. This view is selected by a search process in a solution space for the viewpoints.

Besides techniques for viewpoint selection there are numerous approaches to define a region of interest (ROI) in volumetric data. In the scope of volumes this region is also called volume of interest (VOI). Tory and Swindells [13] presented ExoVis for detail and context direct volume rendering. The VOI can be defined by placing a box within the volume. A translation extracts this part from the volume and this $3 \mathrm{D}$ cutout can be displayed with different rendering styles or transfer functions. Owada et al. [8] presented volume catcher as a technique to specify a ROI within unsegmented volume data. The user defines this region by drawing a $2 \mathrm{D}$ stroke along the contour of the interesting structure and their system performs a constrained segmentation based on statistical region merging. Zhou et al. [20] proposed focal regionguided feature-based volume rendering to emphasize the VOI. In their approach a geometric shape like a sphere is used to divide the volume into a focal and a context region.

Regarding tissue classification interesting research has been done by Sato et al. [10]. They have taken 3D local intensity structures into account to identify local features like edges, sheets, lines and blobs which typically correspond to types of tissue in medical volume data. Their local structure filters use gradient vectors along with the Hessian matrix of the volume intensity combined with Gaussian blurring.

\section{The LiveSync WorkfLOW}

The overall goal of this work is to offer the physician an optimal setup of the viewing parameters for the volumetric view with the least possible effort. If slice views and the volumetric view are not linked the navigation has to be done separately. To enable a 2D/3D synchronization, the functionality of LiveSync can be activated on demand by pressing a hot key while pointing with the mouse on the structure of interest on the slice. Based on this picking process, knowledge-based techniques are applied to estimate good viewpoints for the volumetric view, to calculate an appropriate placement of a view-aligned clipping plane, and to adjust the zoom factor. Depending on the user's preferences, the system allows a smoothly animated rotation or an instant switch between two successive viewpoints. In the case the user is not entirely satisfied with a provided view, it can be refined by manually changing the viewpoint, replacing the clipping-plane, or adjusting the proposed zooming to get a better view of the ROI. If LiveSync is not activated the navigation with the slices is done in a traditional manner and does not lead to an update of the volumetric view. The following factors are considered to achieve the live synchronization:

Picked point: The volumetric position of the depicted structure is determined by the position which the user has picked on a slice.

Slice view zoom: The zoom of the slice view serves as an indicator for the size of the interesting anatomical structure. To set up all viewport parameters automatically this zoom factor is considered to adjust the zoom of the volumetric view. 
Patient orientation: Scanned medical data contain information about the patient's position and orientation. Taking into account knowledge about the performed procedure, a rough estimate of the preferred viewing directions is possible.

Viewpoint history: The last viewpoint is used as a parameter for the selection of the next viewpoint. This means that the system tries to find a good viewpoint close to the last one if this does not counteract the other parameters.

Local shape estimation: The local shape of the picked structure is estimated based on local segmentation. Three major shapes lines, sheets and blobs - are assigned to structures to be utilized as parameters for viewpoint selection.

Visibility: Another parameter is the visibility of the picked structure. To compute visibility, rays are cast from the picked point to a certain number of viewpoints and analyzed regarding occluding structures.

The parameters patient orientation, viewpoint history, local shape estimation and visibility are encoded directly in the viewing spheres. If the particular parameter indicates a good viewpoint at a certain position, a unit sphere is deformed in a way that the distance of this point to the sphere's center is increased. Figure 1 gives an overview on the LiveSync workflow. Initially there is a volumetric view which is shown from a default viewpoint and a $2 \mathrm{D}$ slice view. For each picking action on the slice, the input parameters are used to estimate good viewpoints and to deform the viewing spheres accordingly. This is achieved without any data-specific a priori information and precomputations. The input parameters have to be weighed and combined to get a resulting deformed sphere which encodes the combined quality of the viewpoints. In addition, the zoom factor is adjusted and a viewaligned clipping plane is defined which allows a flexible removal of occluding structures to generate a meaningful visualization.

\section{VieWINg SPHERE}

The viewing sphere and the camera analogies are well-known concepts for setting up a viewpoint and a viewing direction in computer graphics. Basically, a virtual camera can be placed at any point on the surface of a sphere which encapsulates the scene. To move the camera on this sphere typically rotation operations are performed. In addition, the viewing direction of the camera defines on which location in the scene the camera is focusing. Zooming can be achieved by moving the camera along the surface normal of its position on the sphere.

\subsection{Sphere Parameterization}

As the input parameters have to be encoded directly into the sphere's shape there is need for an intuitive way to parameterize the viewing sphere. In addition this parameterization has to be stored efficiently with taking into consideration that operators for the combination of the individual spheres have to be applicable. A convenient parameterization of spheres can be achieved with polar coordinates. In this system each point of a sphere can be characterized by $\theta$ and $\varphi$, which represent the polar and the azimuthal angle, and its radial distance $r$. The polar angle starts from the positive $\mathrm{z}$-axis and ranges from 0 to $180^{\circ}$ and the azimuthal angle in the xy-plane starts from the positive $\mathrm{x}$-axis with a range from 0 to $360^{\circ}$. With this parameterization several conversions and calculations can be computed very efficiently [14, 19].

\subsection{Sphere Map}

A well-known challenge in computer graphics is the problem of applying a texture map to a sphere. The naive approach performs a direct latitude-longitude mapping onto a sphere by using a single rectangular texture in which the width is twice the height. With uv-mapping $u$ spans the equator and $v$ covers the pole-to-pole range. This is a straightforward mapping with the disadvantage that the sampling becomes higher towards the pole regions. Alternatives for spherical textures are cube, omnitect, icosahedral and octahedral mappings [18].

The inverse problem has to be handled to map a sphere to a structure which facilitates the operations that are performed in the presented concept. Because of memory efficiency and intuitive indexing the direct latitude-longitude mapping was the technique of choice. The rectilinear texture is stored as a two-dimensional array with $360 \times 180$ entries. Explicit storing in memory is necessary to facilitate an efficient combination of differently sampled data. In the current implementation information about patient orientation, viewpoint history and local shape estimation is analytically described, whereas visibility information is sampled in a discrete manner. As the angular position can be calculated from the array indices it is sufficient to write the radial distance values to this array.

\subsection{Sphere Deformation}

The general idea to indicate the quality of viewpoints is the direct deformation of the viewing sphere. Positions on the sphere's surface with a high radial distance represent good viewpoints. To achieve an appropriate deformation of the sphere, the Phong illumination model serves as an analogy. In this model a hemisphere represents the diffuse reflection intensity with a bump which indicates the specular reflection intensity. Phong's model of the specular highlight is adapted for the calculation of the radius $r$ at a certain point on the sphere's surface with the equation

$$
r=a \cdot(\mathbf{n} \bullet \mathbf{v})^{m_{w}},
$$

where $a$ is a constant which controls the height of the bump, $\mathbf{n}$ is the surface normal at a specific point on the sphere, $\mathbf{v}$ is the surface normal at a good viewpoint and $m_{w}$ controls the width of the bump. With slight variations of this formula the deformed spheres for most input parameters used for viewpoint selection can be generated.

\section{VieWING-SPHERE MANIPULATORS}

A challenging part in the selection process of a good viewpoint is the identification of the relevant parameters. For a generic solution which works for different types of medical volume data, the definition of objective parameters is important. The patient's orientation, the viewpoint history, the local shape of the structure and its visibility are considered to be of relevance for viewpoint selection. Viewing spheres are deformed to encode the viewpoint quality for each of these components.

\subsection{Patient-orientation viewing-sphere}

The first utilized parameter to construct a deformed viewing sphere is the patient's orientation. According to the type of an examination there exist general preferred viewing directions. In this case the head-feet axis serves as a rough estimation to derive the preferred viewpoints. Figure 2 (left) shows the rotation axis which corresponds to the patient's orientation. The viewing sphere is deformed in a way that it prefers viewpoints which are orthogonal to this axis. This deformation is achieved by applying Equation 1 as it is described in Algorithm 1 where the $\mathrm{z}$-axis is the main rotation axis.
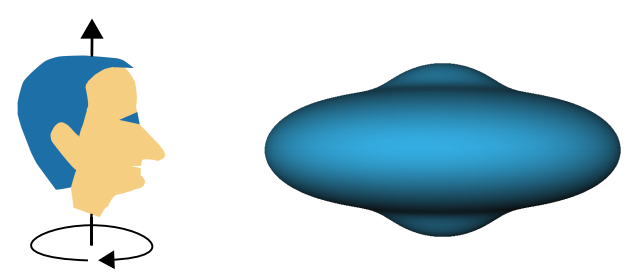

Fig. 2. The head-feet axis is assumed to be the main rotation axis (left). To encode this information in the viewing sphere it is enlarged around the equator (right).

\subsection{Viewpoint-history viewing-sphere}

The selection of a good viewpoint is based on different input parameters to provide the user with an intended view. As a specific view was selected by the system based on estimated demands of the user, the current viewpoint will also be considered for the estimation of the 

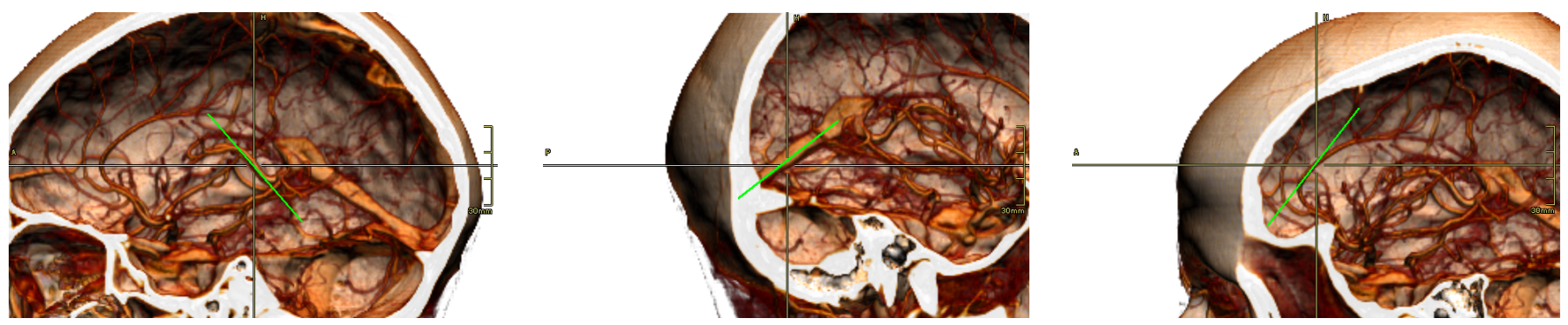

Fig. 3. The green line displays the orientation of the most important feature vector determined by a PCA for three different positions on blood vessels in the head. These vectors are strongly aligned with the local orientation of the vessels.

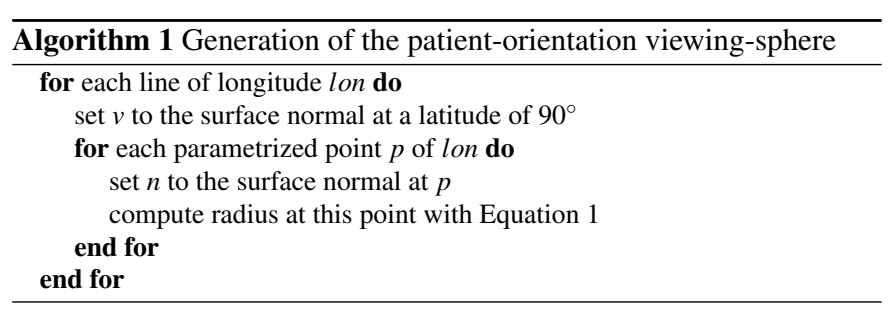

quality of the next viewpoints. Especially, big shifts of the viewpoint for two successive pickings should be avoided if possible. This means that if there is a good viewpoint for the picked structure close to the current one this viewpoint is preferred to others which are positioned farther away on the viewing sphere.
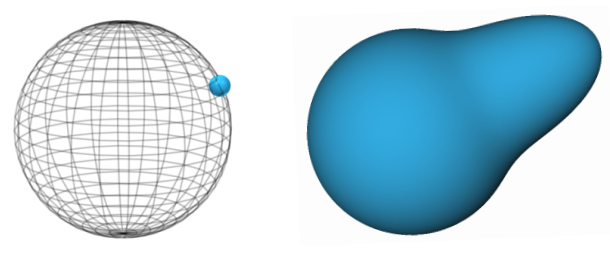

Fig. 4. The marked position on the viewing sphere indicates the last viewpoint (left). The deformed sphere has a single bump which indicates the quality of the viewpoints (right).

Figure 4 shows how the deformed sphere for this criterion should look like. The position of the last viewpoint is marked on the viewing sphere. After deformation the resulting viewing sphere should have a bump with a maximum at this position which also encodes the quality of surrounding viewpoints. The according deformation can be generated with Algorithm 2 .

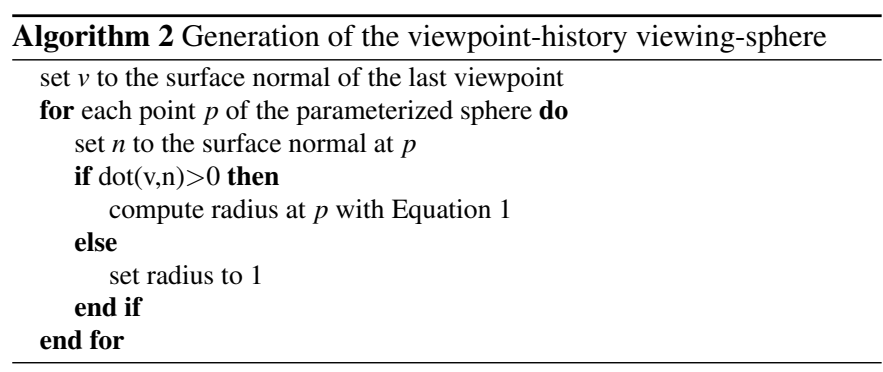

\subsection{Local shape-estimation viewing-sphere}

Another important input parameter for viewpoint selection is the local shape of the structure of interest. If the picked point is, e.g., part of a blood vessel, a good viewpoint shows the course of this vessel and does not cut through it. With a fast local segmentation and a principal component analysis (PCA) the shape information can be derived locally from the data values. Region growing is performed on a $32 \times 32 \times 32$ neighborhood of the picked data point which serves as seed point. The lower and upper threshold for the region growing are calculated by analyzing the distribution of the scalar values at the picked point and its neighborhood. The result of this local segmentation is a connected 3D point cloud. PCA is performed on this point cloud to extract the three feature vectors and the corresponding eigenvalues which are utilized to determine the local feature shape according to a metric of Sato et al. [10]. Figure 3 shows how the vector of the first principal component is oriented when picking is performed at three different positions on blood vessels in the head. The local orientation of the vessels is indicated by these vectors quite well. In combination with the orthogonal second and third principal components and the corresponding eigenvalues this information is used to create the deformed spheres for the local shape estimation.

According to the local shape of the object, the viewing sphere has to be deformed as illustrated in Figure 5. If the object has a volumetric extent (blob), then basically all viewpoints are of the same quality (left). For a planar structure (sheet) the viewpoints which are orthogonal to the sheet are favored (middle). If a tubular structure (line) is determined, the preferred viewpoints are aligned along a ring which is orthogonal to this line (right).
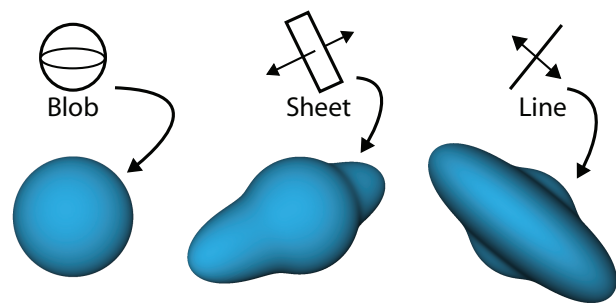

Fig. 5. The viewing sphere which is generated for the local shape estimation is deformed according to the major volumetric extent of the structure.

For the planar object the deformation of the sphere is calculated analogous to the deformed sphere for the viewpoint history. To get two bumps on the opposite sides of the sphere Equation 1 is adjusted slightly to

$$
r=a \cdot a b s\left((\mathbf{n} \bullet \mathbf{v})^{m_{w}}\right) .
$$

If the structure is tubular the deformation process is a bit more complex. It is a generalization of the deformation process of the patientorientation viewing-sphere because the tube can be oriented arbitrarily within the volume. Geometrically the good viewpoints are located around a great circle of the viewing sphere, defined by the two points where the vectors of the second and the third principle components intersect the sphere's surface. A great circle is always uniquely defined by two points on the surface of the sphere and its center is the same as the center of the sphere. For each position $p$ on the sphere's surface the vector from the origin to the closest point on the great circle has to be calculated. This can be achieved by projecting the vector from the origin to $p$ onto the plane of the great circle. The procedure to generate the deformed sphere is presented in Algorithm 3 . 


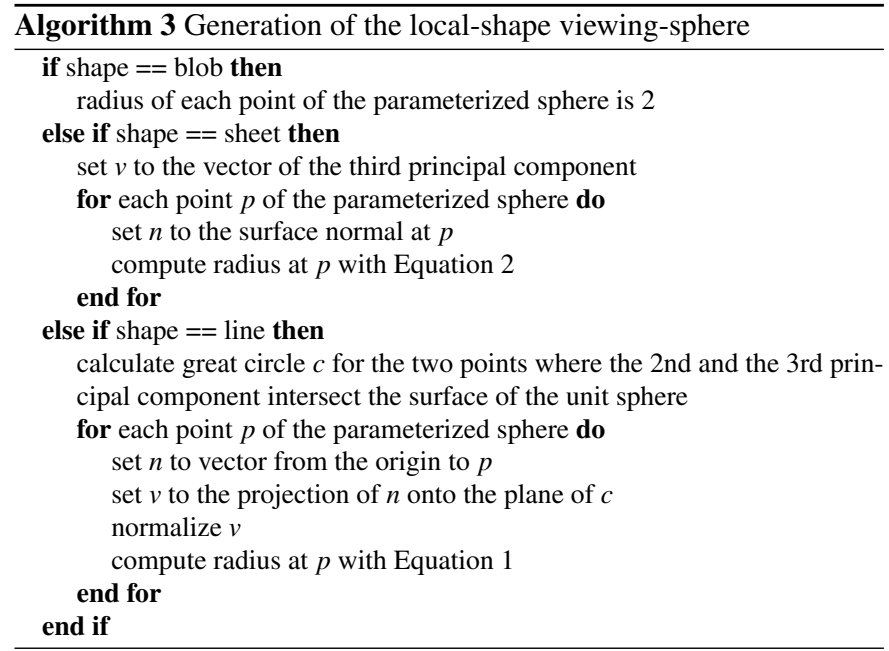

\subsection{Visibility viewing-sphere}

A further building block for estimating a good viewpoint is defined by the visibility information. Starting from the picked point visibility rays are cast to determine occluding objects. As stated in Section 4.1 the parameterized points of the sphere are not distributed uniformly. It is neither efficient nor necessary to cast visibility rays to all $360 \times 180$ positions. Nevertheless it is highly preferable that the positions which are tested are distributed uniformly on the sphere. Bourke [3] provides source code (written by Lettvin) for this purpose. Based on the standard physics formula for charge repulsion an arbitrary number of points is distributed over the surface of a sphere. In our experiments we determined that a subset of $36 \times 18$ rays provides a good trade-off between performance and quality. The calculation of the uniformly distributed points is performed only once and the result is stored in a look-up table.

To determine whether a certain viewpoint provides good visibility of the selected structure, rays are cast from the picked point. As a local segmentation was performed for the local shape estimation, this information is utilized to determine when a ray exits the tissue of interest. When this has happened the opacity information of the transfer function is considered. The opacity is accumulated along the ray and as soon as a small opacity threshold is surpassed the calculation is terminated for the specific ray. A high visibility value is assigned to a viewpoint if there is much space from the picked point in the direction of this viewpoint until it gets occluded by other structures. Such a situation provides more flexibility for positioning the clipping plane. This allows to position the clipping plane orthogonal to the viewing direction far away from the picked point, so that an unobstructed view of the picked point is possible while the helpful context information is not unnecessarily reduced.
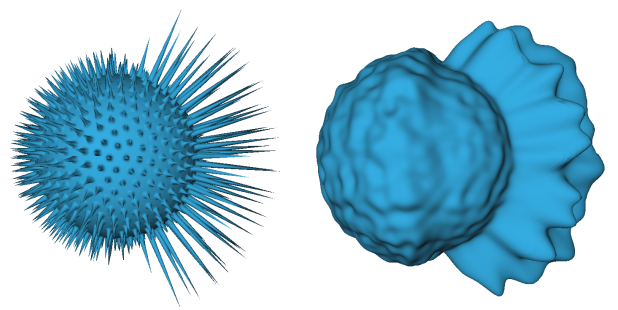

Fig. 6. The lengths of the spikes encode the viewpoint quality at a uniformly distributed set of sample positions (left). After reconstructing at all positions a smooth sphere is generated (right).

The deformed sphere is depicted in Figure 6. One important criterion for the viewpoint entropy of Bordoloi and Shen [2] is view stability which describes the maximum change in a certain view caused by small camera shifts. The view is defined to be stable if a small camera change implies also only small changes in the view. Transfered to the visibility viewing-sphere there is the possibility to encode view stability derived from the visibility values at the discrete uniformly distributed points. It is heuristically assumed that a viewpoint in between several good ones is also rather good. Such a point offers high view stability, because small changes of the viewpoint will also lead to good viewpoints. To encode this information into the viewing sphere, for all the parameterized sphere positions which are not explicitly tested for visibility a weighing with the surrounding tested points is performed. With this weighing a smoothly deformed sphere is obtained as shown in Figure 6 (right). The pseudocode to generate the deformed sphere for the visibility criterion is presented in Algorithm 4 .

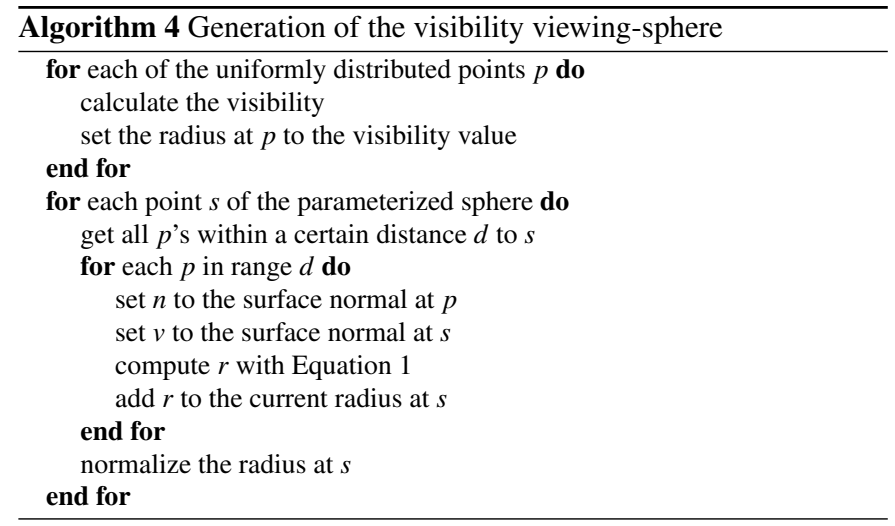

\section{VieWING-Sphere Operators}

After the generation of the deformed viewing spheres for the various view input parameters the question arises how they have to be weighed and how they can be combined to simultaneously accommodate for all the effects.

\subsection{Weighing of viewing spheres}

Equation 1 offers different options to weigh the extent of deformation of a sphere. Basically, $a$ controls the hight of the bump and $m_{w}$ its width. To facilitate the combination operators the values of $a$ for the individual sphere deformations are chosen so that their radii vary from 1 to 2 after deformation. For all the input parameters it makes sense that an estimated good viewpoint also influences the quality of viewpoints in a certain neighborhood. For each viewpoint criterion the radius can vary by a factor of two around a good viewpoint at a certain position. The sphere generation for the viewpoint history contains a built-in weighing control. A big shift of the viewpoint is quite disturbing for picking actions within a small spatial area of the data but it is acceptable for two picked points which are located far apart from each other. This just means that the user is switching to a totally different inspection region whereby the viewpoint coherency is less critical. A distance factor $d$ is calculated as the ratio of the spatial distance between two successively picked points to the diagonal extent of the volume. To influence the weighing for the viewpoint-history viewing-sphere Equation 1 is modified to

$$
r=(1-d) \cdot a \cdot(\mathbf{n} \bullet \mathbf{v})^{m_{w}} .
$$

\subsection{Combination of viewing spheres}

As the deformed spheres were calculated for the input parameters individually, they have to be combined into a single sphere which encodes the overall quality. Currently three operators are implemented for this combination - summation, multiplication and thresholding. Each of these operators emphasizes certain viewpoint characteristics. Figure 7 shows the effects of the three operators on the resulting sphere. For this example the visibility viewing-sphere and the local shapeestimation viewing-sphere are chosen as input spheres. The application of these operators and the development of additional operators is 
easy to achieve because each deformed sphere is parameterized as a two-dimensional array.

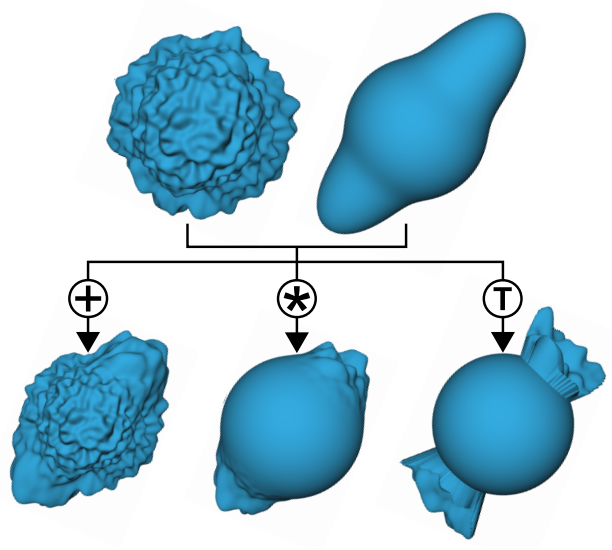

Fig. 7. The currently implemented operators to combine the deformed spheres are summation, multiplication and thresholding.

As operands for the operators, the offset of the radius which is higher than the radius of the unit sphere is taken. At each position the radius of a deformed sphere has a value between 1 and 2 so that the operations are performed on values between 0 and 1 . The implementation and the characteristics of the realized operators are as follows:

Summation: A loop over all entries of the sphere arrays is performed to sum up the corresponding radii. This intuitive approach leads to very good results. Good viewpoints will be detected at positions where at least some of the input spheres indicate a good one. Summation is not as sensitive to outliers as multiplication or thresholding.

Multiplication: To emphasize certain characteristics more strongly an operator is implemented which computes the multiplication of the input spheres. This operator emphasizes positions where good viewpoints are indicated by several source spheres and deemphasizes positions where at least one source sphere indicates a bad viewpoint. Low values have an increased impact on the result. Even if the value of only one input sphere is low the corresponding viewpoint will be rated as a bad one.

Thresholding: For the thresholding operation one specific sphere is taken as the initial one. In a loop over all parameterized points of this sphere the value at this position is only considered if the values of the other spheres at the same position are above a certain threshold. If this is not the case the radius on the specific position is set to 1 . This operator filters out the values where the corresponding values on the other spheres are indicating a bad viewpoint. With the thresholding it is possible to define knockout criteria. Assuming the patient-orientation viewing-sphere is the initial thresholding sphere a window for a certain preferred viewing direction can be defined. By thresholding over the other deformed viewing spheres a good viewpoint within this frame will be estimated.

\section{Derived Viewport Parameters}

After describing the view input parameters, the viewing-sphere manipulators and the viewing-sphere operators, the parameters to set up the volumetric view can be derived. These are the parameters for a good viewpoint, the placement of the view-aligned clipping plane, the zoom and the viewing direction. The application of the viewing-sphere operators to the individual deformed viewing spheres produce the combined viewpoint quality at $360 \times 180$ positions on the joint viewing sphere. A good viewpoint can be easily determined by the highest entry in the sphere-map array which holds the radial distances of all points. The system can then display the data according to the best estimated viewpoint or suggest a small number of preferred views (e.g., displayed as thumbnails).

With the information obtained by the visibility calculation in Section 5.4, the exact position where the picked point is occluded along each tested visibility ray is known. This information is used for setting up of a view-aligned clipping plane to clip away the occluding structures. To position the clipping plane, a location along the ray starting at the picked point where the accumulated opacity is still below a small threshold is selected. This allows an unobstructed view of the picked object while preserving as much context information as possible. The viewing direction is directly defined by the picked point and this point is shown in the center of the volumetric view window. Finally, the zoom factor for this view can be derived from the current settings of the slice view. The zoom of the slice view gives a rough estimation about the size of the interesting anatomical structure. In the current implementation this zoom factor directly determines the zoom of the volumetric view.

\section{Results and Evaluation}

For a convenient evaluation of the results of the LiveSync concept the implementation is integrated into a real world medical workstation which is under development by our collaborating company partner. All the computations for the LiveSync viewpoint selection can be performed interactively and are not influenced significantly by the size of the data set. The performance was measured on a PC configured with an AMD Athlon 64 Dual Core Processor 4400+ and 2 GB of main memory. In the unoptimized implementation, the LiveSyncrelated computations took $70 \mathrm{~ms}$ to $150 \mathrm{~ms}$ per picking, depending on the number of segmented voxels at the local segmentation step and on the estimated local feature-shape. The users get an instant update of the volumetric view whenever they are picking on a certain structure in a $2 \mathrm{D}$ slice. In the remainder of this section, LiveSync-generated images for three different application scenarios and the results of an informal evaluation will be provided to demonstrate the usefulness of the interactively synchronized views.

For the first scenario an estimated good viewpoint and a rather bad viewpoint will be presented for comparison reasons. Figure 8 shows the results for a picking action on a slice. The picking is performed with the aim to get information on the course and the spatial vicinity of the partly visible vessel. A viewpoint which is rated as rather bad by the viewpoint selection process leads to a volumetric view as shown in Figure 8 (right). Important parts of the vessel are occluded, its course remains unclear and the connectivity to other vessels can hardly be revealed by this viewpoint. In Figure 8 (middle), where a good viewpoint defined by the deformed viewing sphere is considered, the information about the vessel's course and its spatial vicinity can be extracted easily. The other two application scenarios demonstrate that LiveSync is a generic tool for various kinds of clinical examinations. In their typical workflow radiologists search for specific structures in medical data. Although there exist highly sophisticated and specialized methods, e.g., for the detection of polyps in the colon or lung nodules, LiveSync can help to quickly explore theses pathological cases. The examination of the colon is a very difficult task with 2D slices only because there it is very hard to see differences between foldings of the colon and polyps. Figure 9 shows the LiveSync result for the picking on a suspicious structure in the colon. With the provided volumetric view it can be clearly seen that the picked structure is not a colon folding but a polyp. Another challenging task is the detection of lung nodules. In the 2D slices they often look very similar to bronchia or vessels. In Figure 10, a structure which is assumed to be a nodule is picked on the slice and LiveSync presents the corresponding volumetric view automatically. This view can clearly help to classify the picked structure as a lung nodule.

In an informal evaluation LiveSync was used by an experienced radiology technician. The used data sets were a head CT angiogram (CTA), a peripheral CTA and a CT of the chest. The goal was to generate 3-4 diagnostically relevant volume renderings of pathologies in the different data sets. This task had to be done at first manually and then 

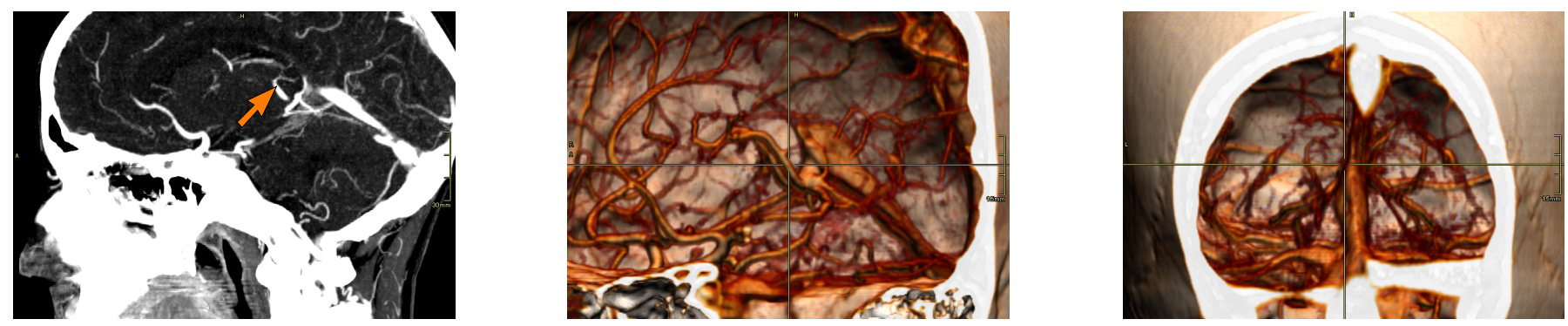

Fig. 8. The picking on the partly visible vessel in a 2D slice image (left). An optimal viewpoint provides useful context information (middle) in contrast to a viewpoint which is estimated as being rather bad (right).

with LiveSync support. In both cases a predefined transfer function was given and the radiology technician had to adjust the other parameters. For all data sets the overall expenditure of time ranged from 5 to $20 \mathrm{~min}$ in the manual case and from 2 to 10 min with LiveSync support. Figure 11 show an exemplary result of the evaluation with the head CTA. To get a diagnostically meaningful view on the aneurysm, the parameters were at first adjusted manually (left). The middle image shows the instant result of just picking a point on a slice. With very little user interaction a clipping plane was adjusted to show an almost identical view (right) to the manually adjusted one. In this informal evaluation the main conclusion was that the effort to localize pathologies diminished extremely when LiveSync was used. The initial views which were suggested with LiveSync support were already very good, but some additional time was needed to adjust them to get screenshots which are perfectly suitable for diagnosis. In the majority of the LiveSync results only small user interaction was necessary to produces screenshots with the same diagnostic value as fully manually adjusted $3 \mathrm{D}$ views. These results are especially satisfying as the radiology technician had no prior experience with the new LiveSync interaction metaphor. The overall impression of the LiveSync feature during the evaluation was that it provides an excellent additional functionality. This opinion is also supported by radiologists getting demonstrations of the LiveSync functionality.

\section{Conclusion And Future Work}

In this paper, we presented a novel concept for the live synchronization of $2 \mathrm{D}$ slice views and a volumetric view within a medical workstation. Different input parameters are identified to generate deformed viewing spheres which indicate the quality of the viewpoints for the specific criteria. After the combination of individual spheres the combined quality of the viewpoints is encoded for $360 \times 180$ parameterized positions on a viewing sphere. Our system provides a good viewport estimation considering patient orientation, viewpoint history, local object shape and visibility. Further it performs an automated placement of a view-aligned clipping plane and zooming. Picking on an interesting structure in the $2 \mathrm{D}$ slice image is the only necessary user interaction to get a meaningful volumetric view. The performed evaluation indicated that the presented approach might considerably improve the efficiency of diagnosis in clinical routine.

In future work we plan to investigate the integration of more information gathered by the user interaction history. With this information it should be possible to generate templates of deformed viewing spheres for different clinical application scenarios. These spheres can provide the physicians with optimal views and optimal view paths for their examinations. Another feature we want to improve on is the automatic set-up of the zoom factor for the volumetric view. We want to utilize the local curvature of the picked object to steer the zooming.

\section{ACKNOWLEDGEMENTS}

The work presented in this paper has been funded by AGFA HealthCare in the scope of the DiagVis project. We would like to thank Rainer Wegenkittl, Lukas Mroz and Matej Mlejnek (AGFA HealthCare) for their collaboration and for providing various CT data sets. Thanks also to Doris Krenn for her great help with the evaluation.

\section{REFERENCES}

[1] V. Blanz, M. J. Tarr, and H. H. Bülthoff. What object attributes determine canonical views? Perception, 28(5):575-599, 1999.

[2] U. D. Bordoloi and H.-W. Shen. View selection for volume rendering. In Proceedings of IEEE Visualization 2005, pages 487-494, 2005.

[3] P. Bourke. Distributing Points on a Sphere. Available online at http://local.wasp.uwa.edu.au/ pbourke/geometry/spherepoints/, March 2007.

[4] M.-Y. Chan, H. Qu, Y. Wu, and H. Zhou. Viewpoint selection for angiographic volume. In Proceedings of the Second International Symposium on Visual Computing 2006, pages 528-537, 2006.

[5] S. Fleishman, D. Cohen-Or, and D. Lischinski. Automatic camera placement for image-based modeling. Computer Graphics Forum, 19(2):101110,2000

[6] C. H. Lee, A. Varshney, and D. W. Jacobs. Mesh saliency. In Proceedings of ACM SIGGRAPH 2005, pages 659-666, 2005.

[7] K. Mühler, M. Neugebauer, C. Tietjen, and B. Preim. Viewpoint selection for intervention planning. In Proceedings of Eurographics/IEEE VGTC Symposium on Visualization 2007, pages 267-274, 2007.

[8] S. Owada, F. Nielsen, and T. Igarashi. Volume catcher. In Proceedings of ACM Symposium on Interactive 3D Graphics and Games 2005, pages 111-116, 2005.

[9] O. Polonsky, G. Patané, S. Biasotti, C. Gotsman, and M. Spagnuolo. What's in an image: Towards the computation of the "best" view of an object. The Visual Computer, 21(8-10):840-847, 2005.

[10] Y. Sato, C.-F. Westin, A. Bhalerao, S. Nakajima, N. Shiraga, S. Tamura, and R. Kikinis. Tissue classification based on 3D local intensity structures for volume rendering. IEEE Transactions on Visualization and Computer Graphics, 6(2):160-180, 2000.

[11] M. Sbert, D. Plemenos, M. Feixas, and F. González. Viewpoint quality: Measures and applications. In Proceedings of Computational Aesthetics in Graphics, Visualization and Imaging 2005, pages 185-192, 2005.

[12] S. Takahashi, I. Fujishiro, Y. Takeshima, and T. Nishita. A feature-driven approach to locating optimal viewpoints for volume visualization. In Proceedings of IEEE Visualization 2005, pages 495-502, 2005.

[13] M. Tory and C. Swindells. Comparing ExoVis, orientation icon, and inplace 3D visualization techniques. In Proceedings of Graphics Interface 2003, pages 57-64, 2003.

[14] J. M. van Verth and L. M. Bishop. Essential Mathematics for Games and Interactive Applications: A Programmer's Guide. Morgan Kaufmann Publishers Inc., San Francisco, CA, USA, 2004.

[15] P.-P. Vázquez, M. Feixas, M. Sbert, and W. Heidrich. Viewpoint selection using viewpoint entropy. In Proceedings of Vision, Modeling, and Visualization 2001, pages 273-280, 2001.

[16] P.-P. Vázquez, M. Feixas, M. Sbert, and W. Heidrich. Automatic view selection using viewpoint entropy and its application to image-based modelling. Computer Graphics Forum, 22(4):689-700, 2003.

[17] I. Viola, M. Feixas, M. Sbert, and M. E. Gröller. Importance-driven focus of attention. IEEE Transactions on Visualization and Computer Graphics, 12(5):933-940, 2006.

[18] Virtual Terrain Project. Spherical Textures. Available online at http://www.vterrain.org/Textures/spherical.html, March 2007.

[19] E. Williams. Aviation Formulary V1.43. Available online at http://williams.best.vwh.net/avform.html, March 2007.

[20] J. Zhou, M. Hinz, and K. D. Tönnies. Focal region-guided feature-based volume rendering. In Proceedings of the 1st International Symposium on $3 D$ Data Processing, Visualization, and Transmission 2002, pages 87-90, 2002. 

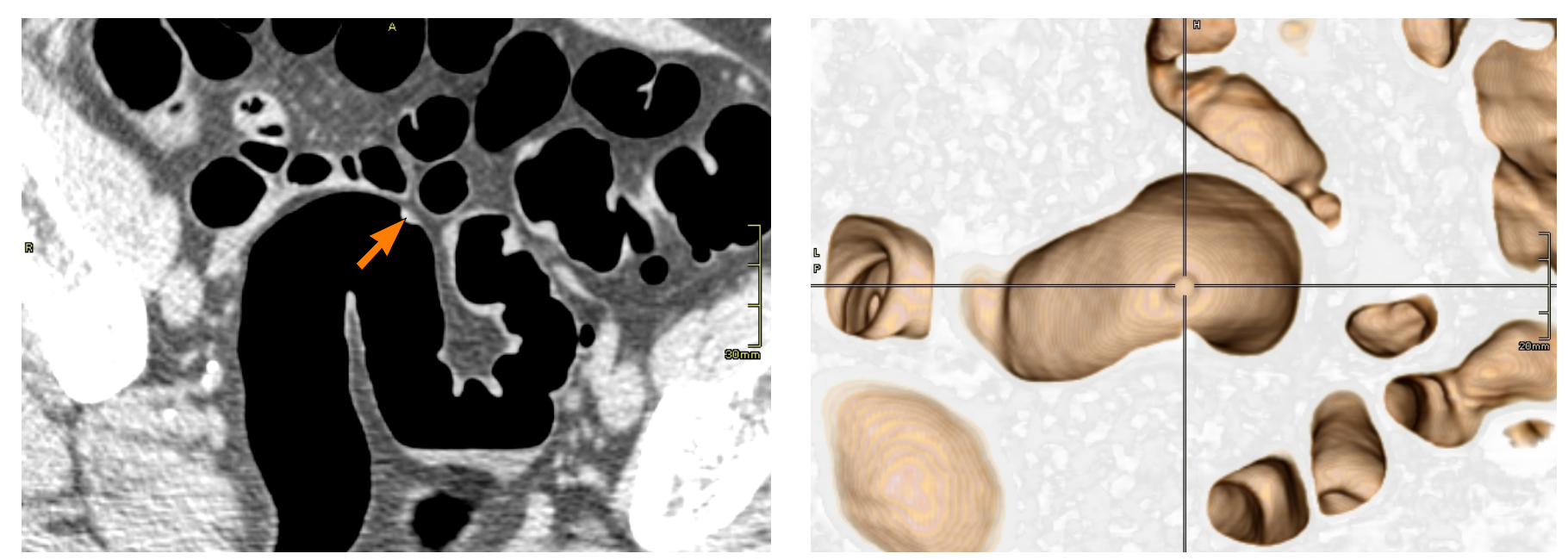

Fig. 9. The picking on a suspicious structure in the $2 \mathrm{D}$ slice of the colon (left) leads to an automatically generated volumetric view (right) in which a polyp can be identified.
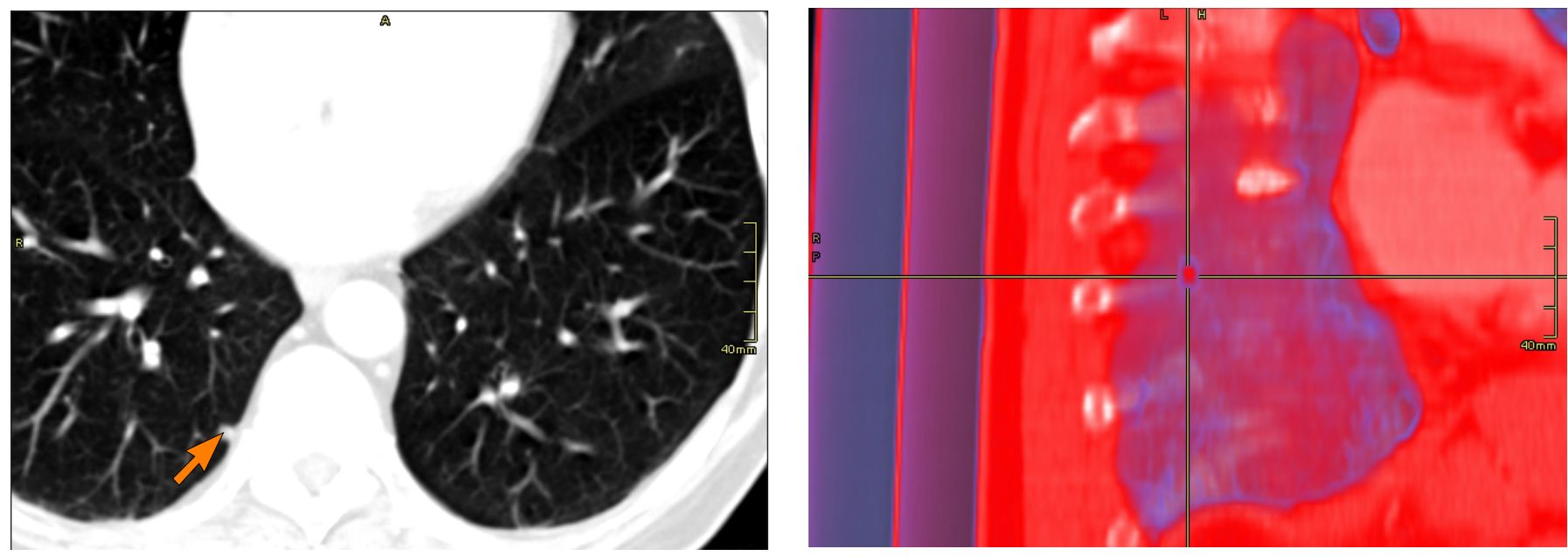

Fig. 10. Lung nodules can be hardly distinguished from vessels or bronchia in the slice view (left). In the volumetric view (right) the picked structure can be clearly classified as a lung nodule.
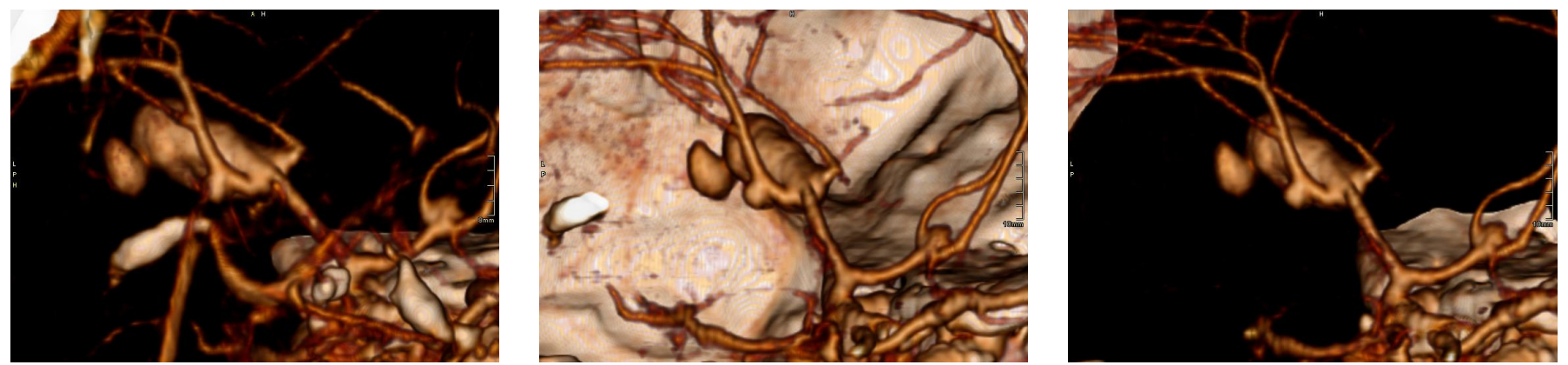

Fig. 11. A manually adjusted image (left) to get a good view on the aneurysm looks very similar to the LiveSync-generated image (middle). After manual clipping (right) the LiveSync-generated image looks almost identical to the manual adjusted image. 